\title{
From Discrete Particles to Continuum Fields in Mixtures
}

\author{
T. Weinhart, S. Luding and A. R. Thornton \\ Multiscale Mechanics, MESA+, Fac. of Eng. Techn., Univ. of Twente, P.O. Box 217, 7500AE Enschede, NL
}

\begin{abstract}
We present a novel way to extract continuum fields from discrete particle systems that is applicable to flowing mixtures as well as boundaries and interfaces. The mass and momentum balance equations for mixed flows are expressed in terms of the partial densities, velocities, stresses and interaction terms for each constituent. Expressions for these variables in terms of the microscopic quantities are derived by coarse-graining the balance equations, and thus satisfy them exactly. A simple physical argument is used to apportion the interaction forces to the constituents. Discrete element simulations of granular chute flows are presented to illustrate the strengths of the new boundary/mixture treatment. We apply the mixture formulation to confirm two assumptions on the segregation dynamics in particle simulations of bidispersed chute flows: Firstly, the large constituent supports a fraction of the stress that is higher than their volume fraction. Secondly, the interaction force between the constituents follows a drag law that causes the large particles to segregate to the surface. Furthermore, smaller particles support disproportionally high kinetic stress, which is a prediction of the theory on shear-induced segregation.
\end{abstract}

Keywords: Coarse graining; Granular flows; Mixtures; Segregation PACS: $47.57 . \mathrm{Gc} ; 45.70 . \mathrm{Ht} ; 83.80 . \mathrm{Fg}$

\section{INTRODUCTION}

Granular flows often contain particles of different sizes, shapes and materials, which can cause the constituent phases to segregate. Under the influence of gravity, kinetic sieving causes the particles to segregate by size, with small particles sifting downwards, as they have a higher probability than large particles to fit into void spaces. Such flows have been described in [1] for bidispersed flows using mixture theory continuum equations.

Discrete particle simulations are a valuable tool to test (and calibrate) continuum models. Therefore, one has to extract macroscopic fields used in the mixture continuum equations from the particle data. Deriving local expressions for these fields is not trivial, especially in highly inhomogeneous regions. It is done here using the coarse-graining approach described in [2], which contains a novel way to describe the interaction between two particle species or with an external boundary. The resulting coarse-grained density, velocity and stress fields $e x$ actly satisfy the momentum equations locally, and an interaction force density (IFD) was defined to describe the interaction with a boundary. The IFD can also be incorporated into the stress, yielding an extended stress definition. The approach has been carefully studied in several publications: In [3], it was shown how to define macroscopic fields that are independent of the coarse-graining width. This approach was successfully applied to flows near boundaries/discontinuities, as well as layered flows [4]. Further, an expression for the displacement gradient was derived in [5], which provides a smooth measure of the shear rate that is accurate even in strongly sheared flows. Here, we apply the approach to mixed flows, and use it to test some of the assumptions of continuum segregation models in bidispersed chute flows.

\section{MIXTURE THEORY}

Here, we review the essentials of mixture theory [6], which can be used to describe granular flows that are composed of different phases. In [1], the theory was used to describe segregation in gravitation-driven chute flows. Here, we use their definitions; the only difference is that we don't assume a scalar stress, but keep the full stress tensor. The granular material is assumed to be a mixture of two constituents, small particles $s$ and large particles l. Partial densities $\rho^{v}$, partial momenta $\rho^{v} \mathbf{v}^{v}$, and partial stresses $\sigma^{v}$ are defined in mixture theory as the share of each constituent $v=s, l$ of the total density $\rho$, momenta $\rho \mathbf{v}$ and stress $\sigma$. Therefore, the sum of the partial values adds up to the total. The IFD, $\beta^{v}$, is the force exerted on phase $v$ by the other constituent, and thus satisfies $\beta^{s}+\beta^{l}=0$. Both phases must satisfy the individual balance laws for mass,

$$
\frac{\partial \rho^{v}}{\partial t}+\nabla \cdot\left(\rho^{v} \mathbf{u}^{v}\right)=0
$$

and momentum,

$$
\frac{\partial \rho^{v} \mathbf{u}^{v}}{\partial t}+\nabla \cdot\left(\rho^{v} \mathbf{u}^{v} \mathbf{u}^{v}\right)=-\nabla \cdot \sigma^{v}+\rho^{v} \mathbf{g}+\beta^{v},
$$

where $\mathbf{a b}$ denotes the dyadic product of two vectors $\mathbf{a}$ and $\mathbf{b}$, and $\mathbf{g}$ denotes the gravitational acceleration vector. 


\section{GRAVITY-DRIVEN SEGREGATION}

Next, we consider flow over a rough, inclined surface, see Fig. 1, and review the kinetic sieving model presented in [1]. We assume that the partial densities and momenta settles to a quasi-steady state much quicker than the flow segregates, such that the temporal derivatives $\partial / \partial t\left(\rho^{v}\right)$ and $\partial / \partial t\left(\rho^{v} \mathbf{u}^{v}\right)$ become negligible after some initial equilibration time $t_{e}$. We further assume that the flow is shallow and thus nearly uniform in flow $(x)$ and crossflow (y) directions. Thus, (1b) simplifies to

$$
0=-\frac{\partial}{\partial z} \sigma_{\alpha z}^{v}+\rho^{v} g_{\alpha}+\beta_{\alpha}^{v}, \quad \alpha=x, y, z, t>t_{e}
$$

Summing up (2) for $v=s, l$ and $\alpha=z$, setting $\left.\sigma_{z z}\right|_{z=\infty}=$ 0 and integrating over $z$ yields the lithostatic balance,

$$
\sigma_{z z}=\rho(h-z) g_{z}
$$

The idea behind kinetic sieving is that the small particles support less of the downward stress than they should according to their volume fraction and thus sink down. To measure this, a stress fraction,

$$
f^{v}=\sigma_{z z}^{v} / \sigma_{z z}
$$

was introduced and assumed to satisfy a functional form

$$
f^{l}=\phi^{l}+B \phi^{s} \phi^{l}, \quad f^{s}=\phi^{s}-B \phi^{s} \phi^{l},
$$

which is chosen such that $f^{s}+f^{l}=1$ and $f^{v}=0$ for $\phi^{v}=0, v=s, l$. According to the kinetic sieving model, we expect the 'overstress' $B$ to be positive, as will be shown later. Gray and Thornton further postulated that the interaction drag can be modelled in analogy with the percolation of fluids through porous solids,

$$
\beta^{v}=\sigma \nabla f^{v}-\rho^{v} c\left(\mathbf{u}^{v}-\mathbf{u}\right) .
$$

Substituting Eqs. (3), (4) and (6) into (2) yields

$$
\phi^{v}\left(w^{v}-w\right)=\left(f^{v}-\phi^{v}\right) \frac{g \cos \theta}{c},
$$

which together with (5) yields

$$
\left(w^{l}-w\right)=q \phi^{s},\left(w^{s}-w\right)=-q \phi^{l},
$$

where

$$
q=\frac{B}{c} g \cos \theta
$$

\section{COARSE-GRAINING}

Next, we provide expressions to compute the partial densities, velocities, stresses, and the interaction force density from discrete particle simulations by applying the coarse-graining approach in [2] to mixtures. We denote the set of walls and fixed wall particles by $\mathscr{W}$, and the phases of small and large particles by $\mathscr{F}^{s}$ and $\mathscr{F}^{l}$, respectively, with $\mathscr{F}=\mathscr{F}^{s} \cup \mathscr{F}^{l}$. Each particle $i$ has mass $m_{i}$, radius $a_{i}$, position $\mathbf{r}_{i}$, velocity $\mathbf{v}_{i}$, as well as rotational degrees of freedom. Each particle pair $i, j$ has contact vector $\mathbf{r}_{i j}=\mathbf{r}_{i}-\mathbf{r}_{j}$, an overlap $\delta_{i j}=\max \left(a_{i}+a_{j}-\right.$ $\left.\left|\mathbf{r}_{i j}\right|, 0\right)$, a contact point $\mathbf{c}_{i j}=\mathbf{r}_{i}+\left(a_{i}-\delta_{i j} / 2\right) \mathbf{r}_{i j}$, and a branch vector $\mathbf{b}_{i j}=\mathbf{r}_{i}-\mathbf{c}_{i j}$. For each constituent $v=s, l$, the partial mass density is defined as

$$
\rho^{v}=\sum_{i \in \mathscr{F} v} m_{i} \phi_{i}
$$

where $\phi_{i}(\mathbf{r}, t)=\phi\left(\mathbf{r}-\mathbf{r}_{i}(t)\right)$ denotes the coarse-graining function. As coarse-graining function, we use a Lucy polynomial [7, 3] with cutoff radius $c$ and width (or standard deviation) $w=c / 2$. To satisfy mass balance, the partial velocity is

$$
\mathbf{V}^{v}=\frac{\mathbf{p}^{v}}{\rho^{v}}, \text { with } \mathbf{p}^{v}=\sum_{i \in \mathscr{F} v} m_{i} \mathbf{v}_{i} \phi_{i} .
$$

The partial stress is the sum of the partial kinetic stress,

$$
\sigma^{k, v}=\sum_{i \in \mathscr{F} v} m_{i} \mathbf{V}_{i}^{\prime} \mathbf{V}_{i}^{\prime} \phi_{i}
$$

with $\mathbf{V}_{i}^{\prime}(\mathbf{r}, t)=\mathbf{V}(\mathbf{r}, t)-\mathbf{v}_{i}(t)$ the fluctuating velocity, and the partial contact stress,

$$
\begin{aligned}
\sigma^{c, v} & =\sum_{i, j \in \mathscr{F} v} \mathbf{f}_{i j} \mathbf{b}_{i j} \psi_{i j}+\sum_{i \in \mathscr{F} v, j \in \mathscr{F} / \mathscr{F} v} \mathbf{f}_{i j} \mathbf{b}_{i j} \psi_{i j} \\
& +\sum_{i \in \mathscr{F} v, j \in \mathscr{W}} \mathbf{f}_{i j} \mathbf{b}_{i j} \psi_{i j}^{\infty} .
\end{aligned}
$$

with $\psi_{i j}(\mathbf{r}, t)=\int_{0}^{1} \phi\left(\mathbf{r}-\mathbf{r}_{i}(t)+s \mathbf{b}_{i j}(t)\right) d s$ the integral of the coarse-graining function over the branch vector and $\psi_{i j}^{\infty}(\mathbf{r}, t)=\int_{0}^{\infty} \phi\left(\mathbf{r}-\mathbf{r}_{i}(t)+s \mathbf{b}_{i j}(t)\right) d s$ the integral over the branch vector extended into the basal surface (see the extended stress definition in [2] for details). Finally, the interaction force density acting on the constituent $v$ is

$$
\beta^{v}(\mathbf{r}, t)=\sum_{i \in \mathscr{F} v} \sum_{j \in \mathscr{F} / \mathscr{F} v} \mathbf{f}_{i j}(t) \phi\left(\mathbf{r}-\mathbf{c}_{i j}(t)\right) .
$$

Note that we distribute the stresses in (13) according to the share of the contact line (i.e., the branch vector) contained in each particle. The idea behind this is that the particles are transferring momentum from their centre of mass to the contact point, thus the smaller particle has a smaller share of the stress. Furthermore, for a collinear collision between two particles $i$ and $j$, the contact force on particle $i$ is independent of the size of particle $j$, so the stress contribution to particle $i$ should not depend on the size of particle $j$. Equation (13) satisfies this basic physical principle. The contribution of the interspecies collisions to the partial stresses is important, as it has a large effect on the fitting of (5). 


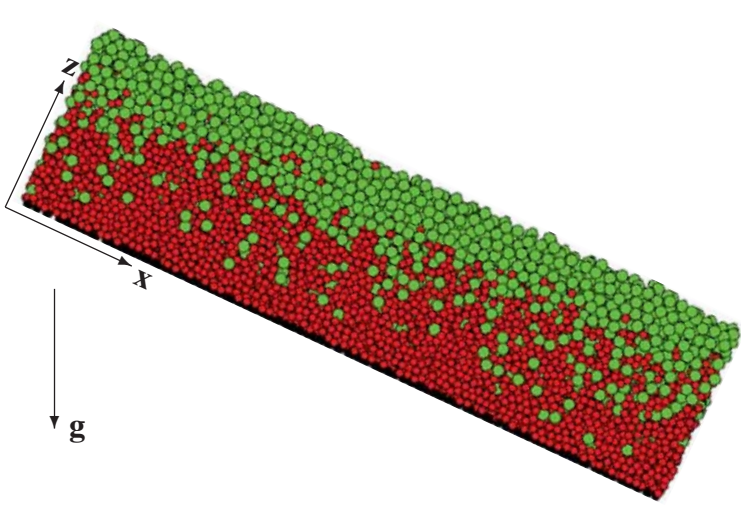

FIGURE 1. Snapshot of the simulation at $t=100 \mathrm{~s}$; Colors/shades indicate fixed (black), large (green/light) and small (red/dark) particles.

\section{SETUP}

A bidispersed flow of particles down a rough inclined surface is simulated, where $x$ is the downslope direction, $y$ the cross-slope direction and $z$ is the direction normal to the basal surface. Figure 1 shows a snapshot of the final state at $t=100 \mathrm{~s}$. The simulations take place in a three-dimensional box, which is periodic in $x$ and $y, 5 d_{s}$ wide, $83.3 d_{s}$ long and inclined at an angle of $25^{\circ}$. The base was created by fixing small particles randomly to a flat surface. The flow is bidispersed, with a size ratio of $d_{l} / d_{s}=1.5$. The simulations are performed with 5000 flowing small particles and 1481 large particles such that the total volumes of large and small particles are equal. Initially, the flow is a homogeneous mixture of randomly distributed particles. Simulation parameters were chosen based on particles with $\rho_{p}=2400 \mathrm{~kg} / \mathrm{m}^{3}, g=9.81 \mathrm{~m} / \mathrm{s}^{2}$ and $d_{s}=0,6 \mathrm{~mm}$. A linear spring-dashpot model is used with contact duration $t_{c}=0.005 \sqrt{d_{s} / g}$, restitution coefficient $r_{c}=0.6$ and contact friction coefficient $\mu_{c}=0.8$. More details about the simulation are available in [8].

\section{RESULTS}

The flow quickly settles to a quasi-steady state at $t_{e} \approx 2.5 \mathrm{~s}$, after which the density, velocity, and stress profiles change only slowly. After equilibration, the large particles segregate upwards, yielding a monotonously increasing volume fraction $\phi^{l}(z)$ that becomes steeper over time (see [8] for a graph of the steady state volume fraction). The segregation is complete and all macroscopic variables are steady after $t \approx 30 \mathrm{~s}$.

We will first study the steady state flow and analyse the time-dependent segregation process later. To obtain statistics for the steady state, the data is averaged in both $x$ and $y$, using a coarse-graining width of $w=d_{s}$, and over the interval $t \in[60 \mathrm{~s}, 100 \mathrm{~s}]$, using snapshots in time at a rate of $0.01 s$. The stress fraction $f^{v}$ almost equals

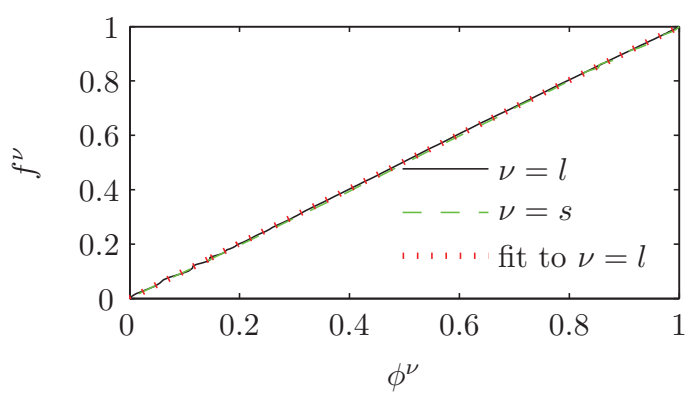

FIGURE 2. Stress fraction $f^{v}=\sigma_{z z}^{v} / \sigma_{z z}$ in steady state as a function of volume fraction $\phi^{v}=\rho^{v} / \rho$, for each constituent $v=s, l$ and fit $f^{l, f i t}$ for $B=0.02$.

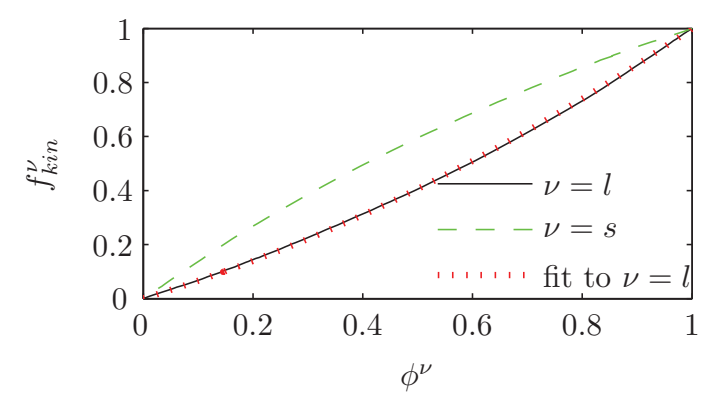

FIGURE 3. Kinetic stress fraction $f^{v}=\sigma_{z z}^{k, v} / \sigma_{z z}^{k}$ in steady state as a function of volume fraction $\phi^{v}=\rho^{v} / \rho$, for each constituent $v=s, l$ and fit $f^{l, f i t}$ for $B=-0.38$.

the volume fraction (see Fig. 2), but the stress fraction of large particles is in fact slightly higher than their volume fraction. This is confirmed by fitting (5) such that

$$
B=\frac{\int_{0}^{1}\left(f^{l}-\phi^{l}\right) \mathrm{d} \phi^{l}}{\int_{0}^{1}\left(\phi^{s} \phi^{l}\right) \mathrm{d} \phi^{l}},
$$

which yields a slightly positive value of $B=0.02$. This value is smaller than what has been shown in [9] for polydispersed flows. Note that the fitting parameter $B$ depends strongly on the way the interspecies stress is distributed. If contact stress is split equally between the small and large particles, $B \approx 0.1$.

The kinetic stress fraction, $f_{\text {kin }}^{v}=\sigma_{z z}^{k, v} / \sigma_{z z}^{k}$, is plotted in Fig. 3 and fitted to (5), which yields a large negative value $B_{k i n}=-0.38$. Thus, the smaller particles support a kinetic stress fraction higher than their volume fraction. This is in agreement with the theory of shear-induced segregation of Fan and Hill [10], which states that the small particles support a higher kinetic stress and thus segregate towards regions of high shear.

Next, we study the segregation over time. To obtain smooth transient statistics, time-averaging is done over intervals of $[t, t+1 \mathrm{~s}]$ for $t=0 \mathrm{~s}, 1 \mathrm{~s}, \ldots, 40 \mathrm{~s}$. Figure 4 shows the upwards movement of the large particles, which starts at $t=0 \mathrm{~s}$ and slowly decreases in strength. The overstress $B$, plotted in Fig. 5, is positive after equilibration, $t \geq t_{e}$, and quickly decreases to a small positive 


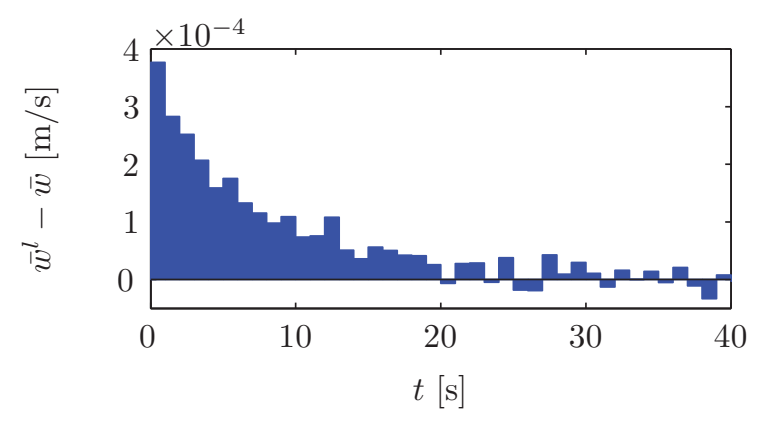

FIGURE 4. Mean upwards velocity of the large particles relative to the bulk, $\bar{w}-\bar{w}^{l}$, over time $t$.

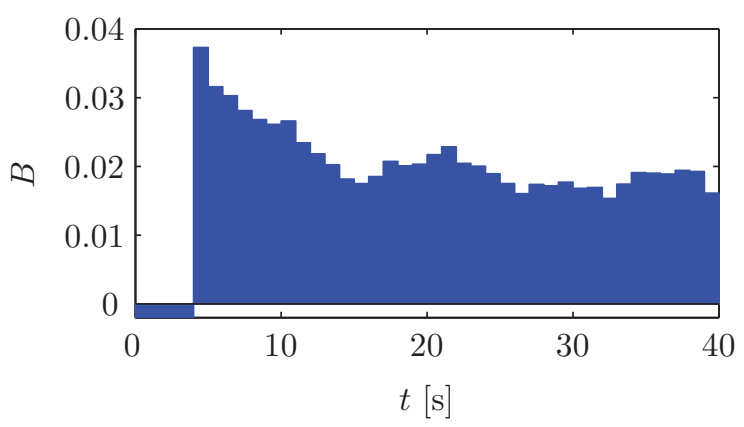

FIGURE 5. Magnitude of overstress, $B$, over time $t$.

steady state value, $B=0.02$. We further fit the interparticle drag coefficient $c$ in (6) using

$$
c=\frac{\int_{\mathbb{R}}\left(\sigma \nabla f^{v}-\beta^{v}\right) \mathrm{d} z}{\int_{\mathbb{R}}\left(\rho^{v} c\left(\mathbf{u}^{v}-\mathbf{u}\right)\right) \mathrm{d} z} .
$$

We compute the segregation rate $q$ by substituting the fits (15) and (16) into (9) and plot the results in Fig. 6. The segregation rate $q$ and the mean upwards velocity of the large particles relative to the bulk, $\bar{w}-\bar{w}^{l}$, show good agreement with (8).

\section{CONCLUSION}

We presented expressions to calculate the partial densities, velocities, stresses and interaction force densities for discrete particle systems with two distinct particle species. The derivation follows the novel coarsegraining approach developed in [2] for boundary interactions, yielding expressions that exactly satisfy the mass and momentum balance, even locally. While it is trivial to define the partial density and velocity, it is not obvious how to define the partial stresses. A simple physical argument is used to correctly apportion the interaction forces to the constituents. These expressions are then applied to measure segregation dynamics in discrete particle simulations of bidispersed chute flows. We test the kinetic sieving model of Gray and Thornton [1]. Fitting the stress fraction to (5) yields a small positive overstress approaching $B=0.02$ as the flow segregates. This confirms

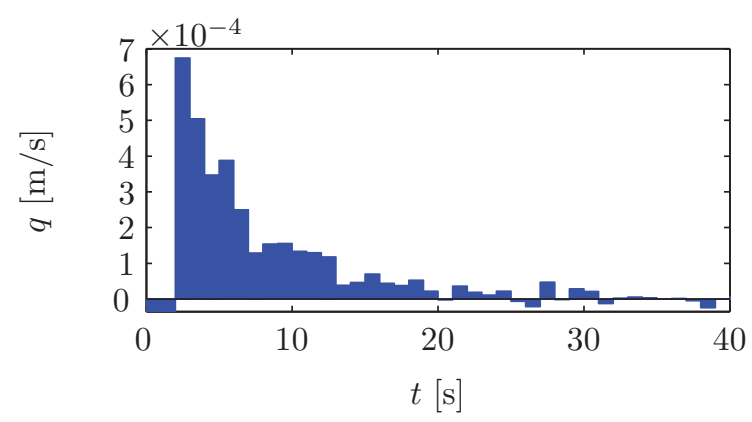

FIGURE 6. Mean segregation velocity $q=(B / c) g_{z}$ over time $t$.

that the large particles support a fraction of the stress that is higher than their volume fraction. The interaction force roughly follows a drag law established in [1], that causes the large particles to segregate towards the surface. The drag coefficient $c$ gradually reduces to zero as the flow reaches a steady state, proving that a simple linear drag law is not sufficient to describe the flow behaviour. Further, the small particles support a fraction of the kinetic stress that is higher than their volume fraction, as postulated in the shear-induced segregation theory of Fan and Hill [10]. To ensure that results shown here for the size ratio $d_{l} / d_{s}=1.5$ are reproducible, all fittings have been repeated for $d_{l} / d_{s}=2.0$; similar fittings were found. The results presented here demonstrate that our new coarsegraining approach is well-suited to study segregation and other mixed-flow phenomena with high local accuracy. An extensive study on the gravity-induced segregation using this new tool is in progress.

\section{ACKNOWLEDGMENTS}

We acknowledge financial support by DFG PiKo 1486, NWO STW VICI 10828 and IMPACT-SIP1. Particle simulations and coarse-graining were undertaken using MercuryDPM.

\section{REFERENCES}

1. J. M. N. T. Gray, and A. R. Thornton, Proc. Royal Soc. A 461, 1447-1473 (2005).

2. T. Weinhart, A. Thornton, S. Luding, and O. Bokhove, Granul. Matter 14, 289-294 (2012).

3. T. Weinhart, R. Hartkamp, A. R. Thornton, and S. Luding, Phys. Fluids submitted (2012).

4. T. Weinhart, A. Thornton, S. Luding, and O. Bokhove, Granul. Matter 14, 531-552 (2012).

5. R. Hartkamp, A. Ghosh, T. Weinhart, and S. Luding, J. Chem. Phys. 137, 044711 (2012).

6. L. Morland, Survey in Geophysics 13, 209-268 (1992).

7. L. Lucy, Astron. J. 82, 2261924 (1977).

8. A. Thornton, T. Weinhart, S. Luding, and O. Bokhove, Int. J. Modern Physics C 23 (2012).

9. B. Marks, P. Rognon, and I. Einav, J. Fluid Mech. 690 (2012).

10. Y. Fan, and K. M. Hill, New Journal of Physics 13 (2011). 